\title{
Bromine Incorporation and Suppressed Cation Rotation in Mixed- Halide Perovskites
}

Andrew Johnston ${ }^{1}$, Grant Walters ${ }^{1}$, Makhsud I. Saidaminov ${ }^{1,2}$, Ziru Huang ${ }^{1}$, Koen Bertens ${ }^{1}$, Niina Jalarvo $^{3}$, Edward H. Sargent ${ }^{1 *}$

${ }^{I}$ Department of Electrical and Computer Engineering, University of Toronto, Toronto, Ontario, Canada

${ }^{2}$ Department of Chemistry and Electrical \& Computer Engineering, Centre for Advanced Materials and Related Technologies (CAMTEC), University of Victoria, Victoria

${ }^{3}$ Oak Ridge National Laboratory, Oak Ridge, TN, USA

\section{Supporting Information}

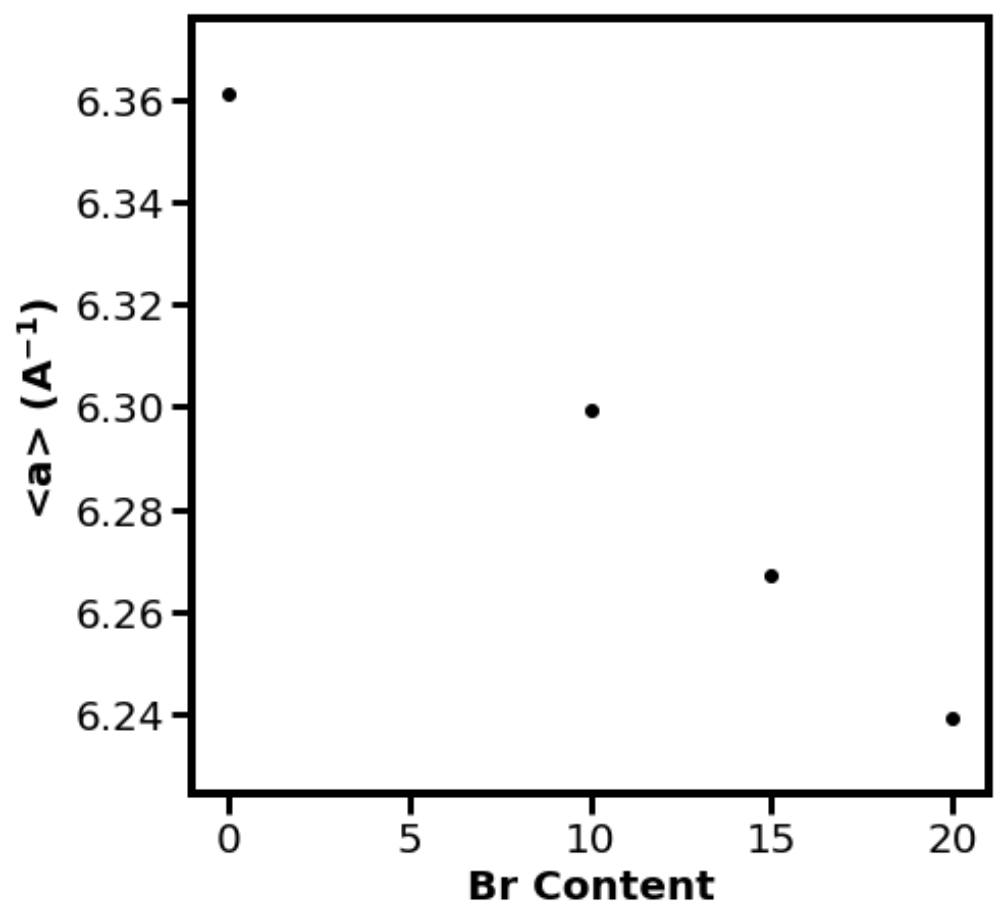

Figure S1: The calculated lattice constant of each single crystal as a function of bromine incorporation. 


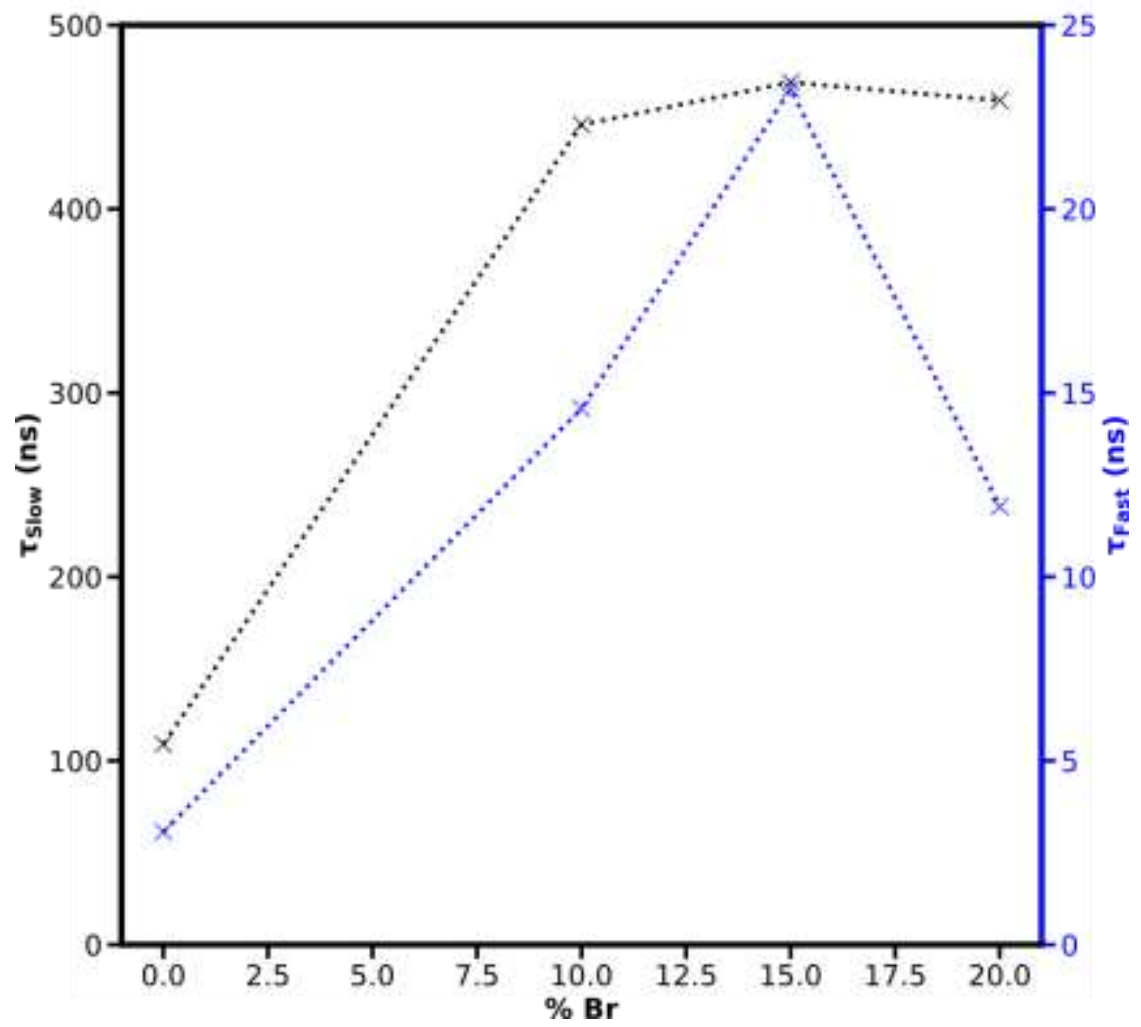

Figure S2. The slow and fast components of the PL-decay of the single crystal samples measured at room temperature.

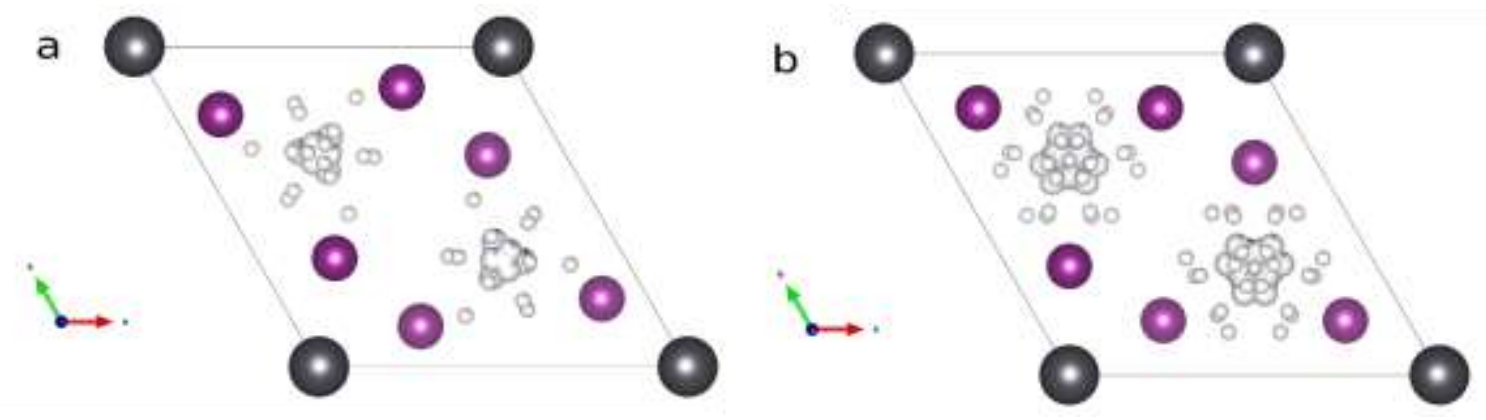

Figure S3. The crystal structures of $\mathrm{FAPbI}_{3}$ at a) $15 \mathrm{~K}$ and b) $220 \mathrm{~K}$. Reproduced from tabulated crystal structure data ${ }^{1}$ 


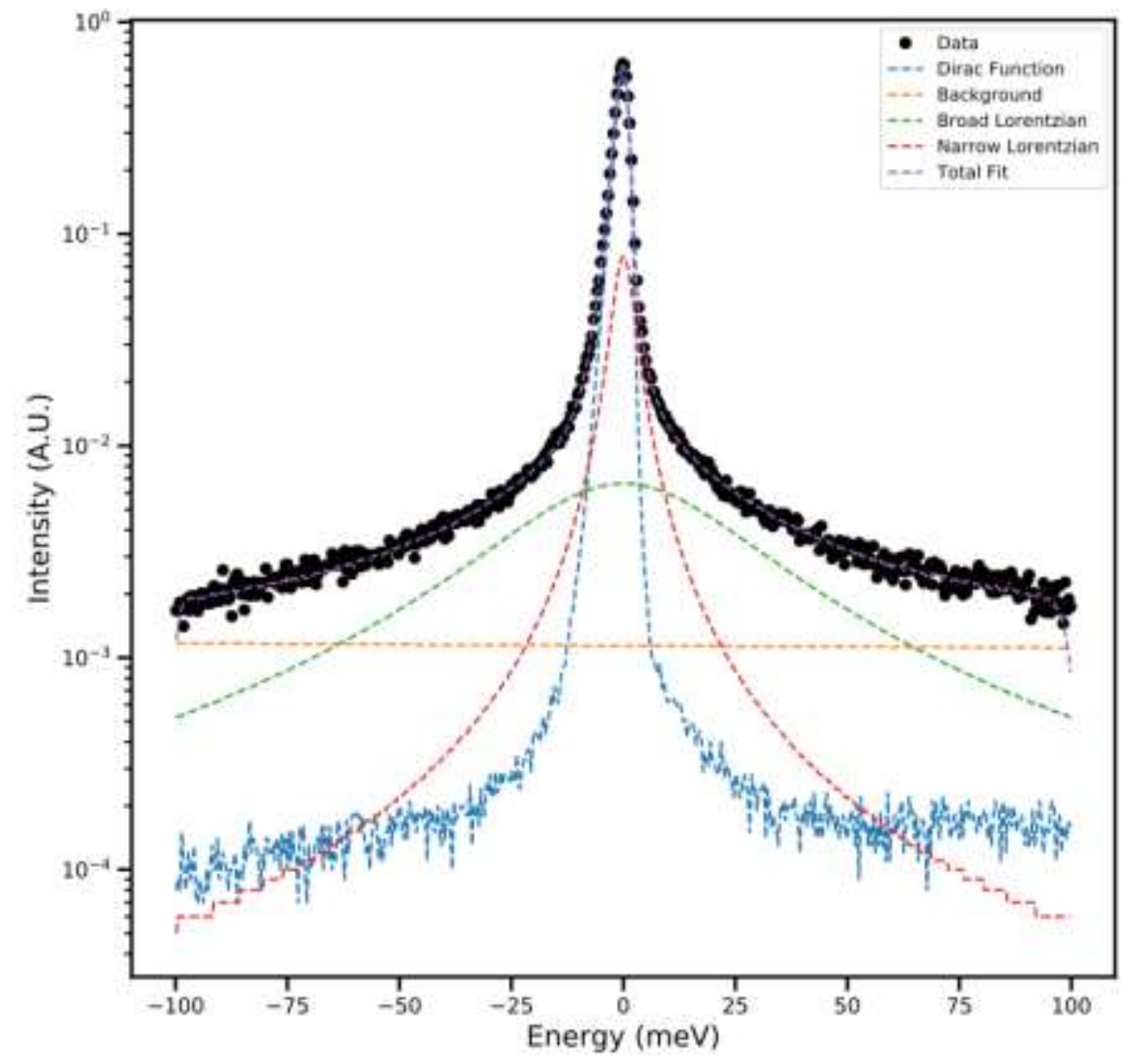

Figure S4. The fitting of the broadened quasielastic neutron signal. Each sample at each temperature was fit with a delta function, a flat background and two Lorentzian functions, each of which excepting the background were convolved with the instrument resolution function. The full-width at half maximum (FWHM) of the Lorentzian functions were kept fixed across each momentum (Q) bin. Each measurement had 9 Q bins. 

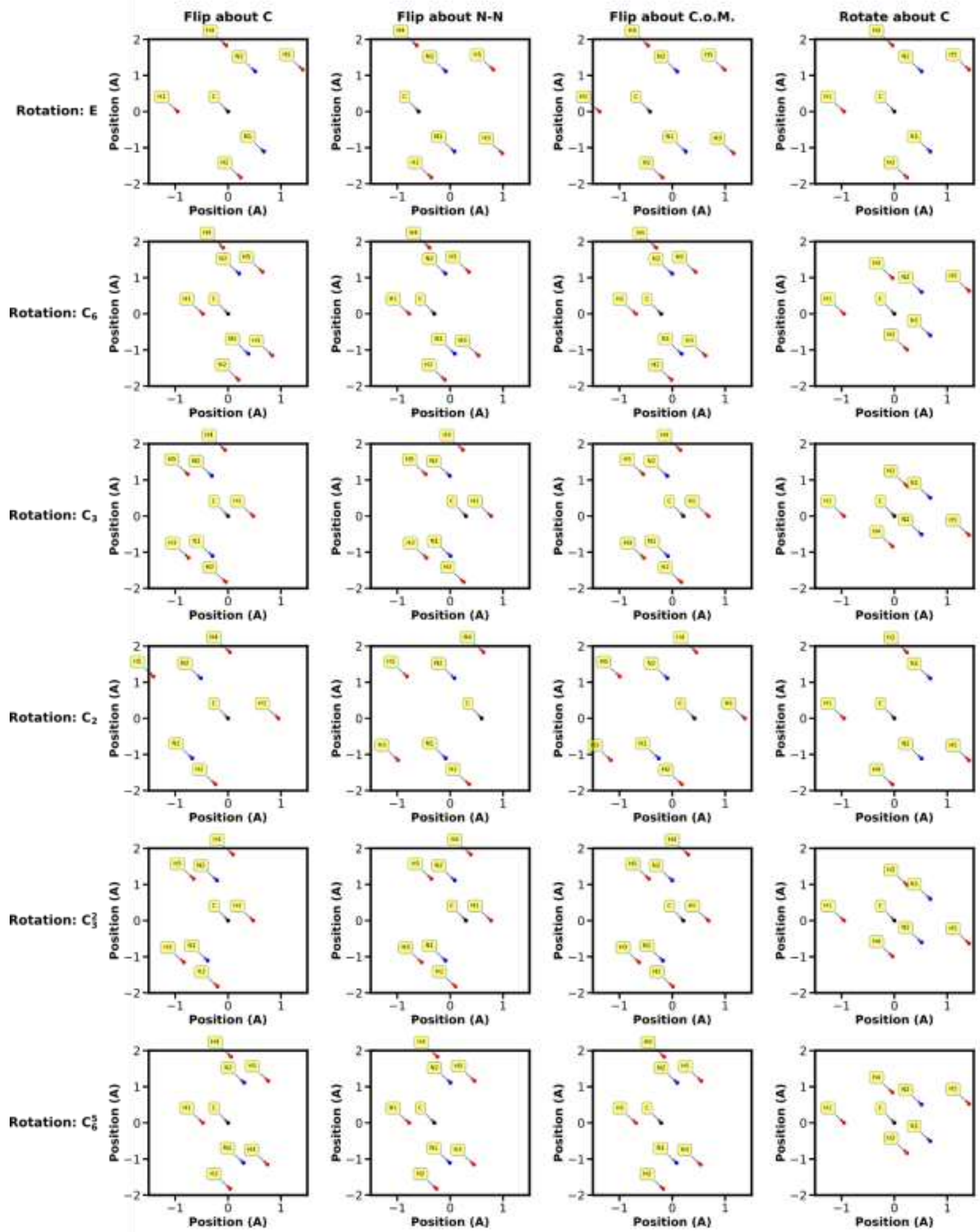

Figure S5. Two-dimensional representations of all FA rotations studied. 
Table S1. The distances travelled by each hydrogen atom under the possible FA rotations (in A).

Flip about C

\begin{tabular}{|l|l|l|l|l|l|}
\hline & H1 & H2 & H3 & H4 & H5 \\
\hline $\mathbf{E}$ & 0.00 & 0.00 & 0.00 & 0.00 & 0.00 \\
\hline $\mathbf{C}_{\mathbf{6}}$ & 0.95 & 0.26 & 1.58 & 0.09 & 1.41 \\
\hline $\mathbf{C}_{\mathbf{3}}$ & 1.65 & 0.44 & 2.74 & 0.16 & 2.45 \\
\hline $\mathbf{C}_{2}$ & 1.90 & 0.51 & 3.17 & 0.19 & 2.83 \\
\hline $\mathbf{C}_{\mathbf{3}}{ }^{2}$ & 1.65 & 0.44 & 2.74 & 0.16 & 2.45 \\
\hline $\mathbf{C}_{\mathbf{6}}{ }^{5}$ & 0.95 & 0.26 & 1.58 & 0.09 & 1.41 \\
\hline
\end{tabular}

Flip about Center of Mass

\begin{tabular}{|l|l|l|l|l|l|}
\hline & H1 & H2 & H3 & H4 & H5 \\
\hline $\mathbf{E}$ & 0.00 & 0.00 & 0.00 & 0.00 & 0.00 \\
\hline $\mathbf{C}_{\mathbf{6}}$ & 1.37 & 0.20 & 1.16 & 0.46 & 0.99 \\
\hline $\mathbf{C}_{3}$ & 2.38 & 0.35 & 2.01 & 0.80 & 1.72 \\
\hline $\mathbf{C}_{2}$ & 2.75 & 0.41 & 2.33 & 0.93 & 1.99 \\
\hline $\mathbf{C}^{2}$ & 2.38 & 0.35 & 2.01 & 0.80 & 1.72 \\
\hline $\mathbf{C}_{\mathbf{6}}{ }^{\mathbf{5}}$ & 1.37 & 0.20 & 1.16 & 0.46 & 0.99 \\
\hline
\end{tabular}

Flip about N-N axis

\begin{tabular}{|l|l|l|l|l|l|}
\hline & H1 & H2 & H3 & H4 & H5 \\
\hline $\mathbf{E}$ & 0.00 & 0.00 & 0.00 & 0.00 & 0.00 \\
\hline $\mathbf{C}_{\mathbf{6}}$ & 1.55 & 0.37 & 0.99 & 0.64 & 0.82 \\
\hline $\mathbf{C}_{3}$ & 2.68 & 0.64 & 1.71 & 1.10 & 1.42 \\
\hline $\mathbf{C}_{2}$ & 3.10 & 0.74 & 1.98 & 1.27 & 1.64 \\
\hline $\mathbf{C}^{2}{ }^{2}$ & 2.68 & 0.64 & 1.71 & 1.10 & 1.42 \\
\hline $\mathbf{C}_{\mathbf{6}}{ }^{\mathbf{5}}$ & 1.55 & 0.37 & 0.99 & 0.64 & 0.82 \\
\hline
\end{tabular}

Twirl about C

\begin{tabular}{|l|l|l|l|l|l|}
\hline & H1 & H2 & H3 & H4 & H5 \\
\hline $\mathbf{E}$ & 0.00 & 0.00 & 0.00 & 0.00 & 0.00 \\
\hline $\mathbf{C}_{\mathbf{6}}$ & 0.00 & 1.83 & 1.16 & 1.83 & 1.16 \\
\hline $\mathbf{C}_{\mathbf{3}}$ & 0.00 & 3.17 & 2.00 & 3.18 & 2.01 \\
\hline $\mathbf{C}_{\mathbf{2}}$ & 0.00 & 3.66 & 2.31 & 3.67 & 2.32 \\
\hline $\mathbf{C}^{2}$ & 0.00 & 3.17 & 2.00 & 3.18 & 2.01 \\
\hline $\mathbf{C}_{\mathbf{6}}{ }^{\mathbf{5}}$ & 0.00 & 1.83 & 1.16 & 1.83 & 1.16 \\
\hline
\end{tabular}



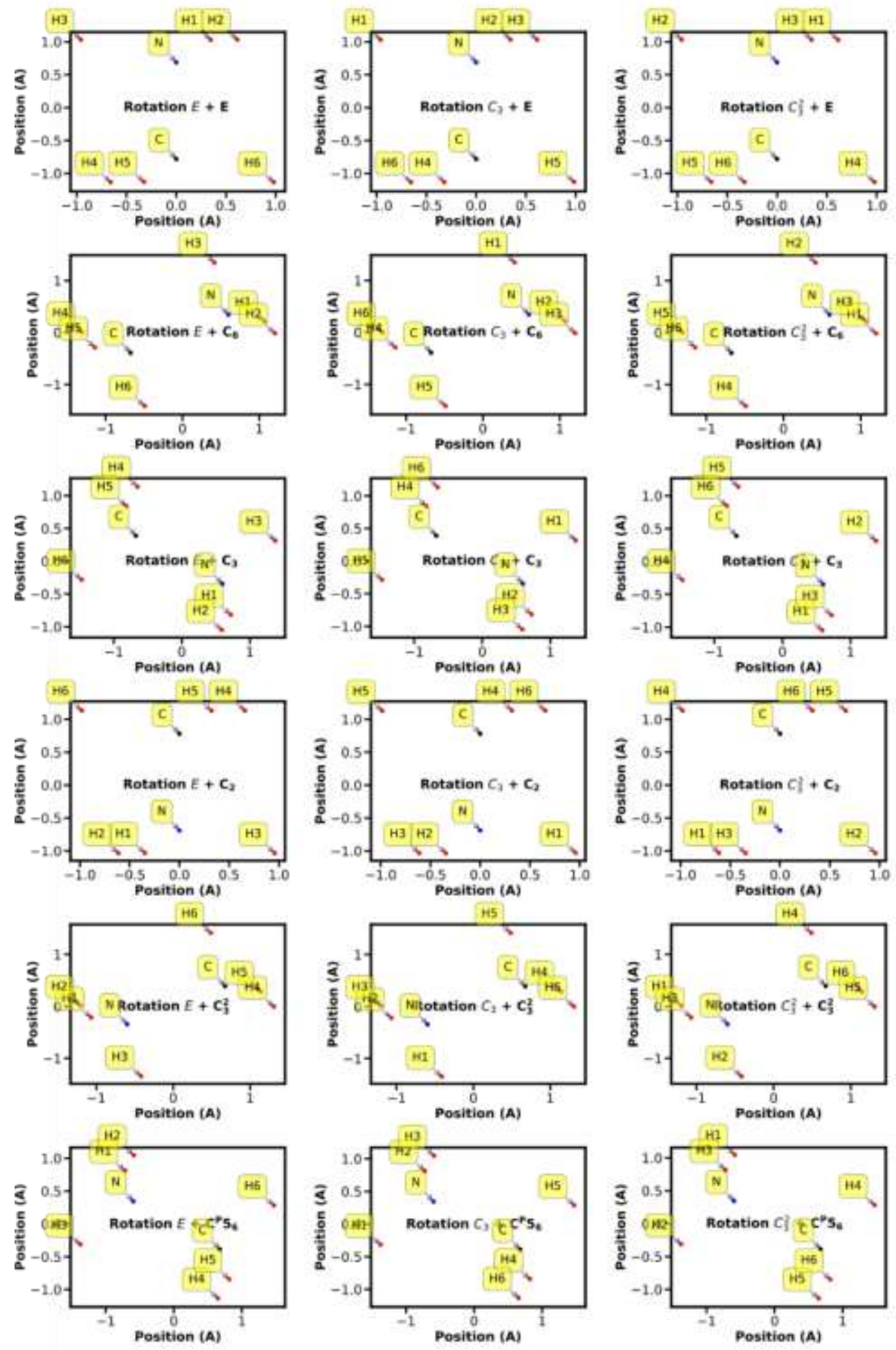

Figure S6. Two-dimensional representation of all rotations under the joint $\mathrm{C}_{3} \otimes \mathrm{C}_{6}$ rotation for MA. 
Table S2. The distances travelled by each hydrogen atom under the possible MA rotations (in A).

\begin{tabular}{|c|c|c|c|c|c|c|}
\hline & H1 & H2 & H3 & H4 & H5 & H6 \\
\hline $\mathbf{E} \times \mathbf{E}$ & 0.00 & 0.00 & 0.00 & 0.00 & 0.00 & 0.00 \\
\hline$E \times C_{6}$ & 1.09 & 1.20 & 1.41 & 1.31 & 1.17 & 1.50 \\
\hline $\mathrm{E}_{\mathrm{X} \mathrm{C}}$ & 1.88 & 2.08 & 2.44 & 2.27 & 2.03 & 2.59 \\
\hline $\mathrm{E} \times \mathrm{C}_{2}$ & 2.17 & 2.40 & 2.82 & 2.62 & 2.35 & 2.99 \\
\hline $\mathrm{E} \times \mathrm{C3}^{2}$ & 1.88 & 2.08 & 2.44 & 2.27 & 2.03 & 2.59 \\
\hline $\mathrm{E} \times \mathrm{C}_{6}{ }^{5}$ & 1.09 & 1.20 & 1.41 & 1.31 & 1.17 & 1.50 \\
\hline$C_{3} \times E$ & 1.68 & 1.68 & 1.68 & 1.73 & 1.74 & 1.73 \\
\hline $\mathrm{C}_{3} \times \mathrm{C}_{6}$ & 1.11 & 1.90 & 2.47 & 1.95 & 1.19 & 2.61 \\
\hline $\mathrm{C}_{3} \times \mathrm{C}_{3}$ & 1.64 & 2.49 & 2.66 & 2.61 & 1.82 & 2.85 \\
\hline $\mathrm{C}_{3} \times \mathrm{C}_{2}$ & 2.40 & 2.82 & 2.18 & 2.99 & 2.62 & 2.36 \\
\hline $\mathrm{C}_{3} \times \mathrm{C}_{3}{ }^{2}$ & 2.71 & 2.67 & 1.21 & 2.85 & 2.91 & 1.31 \\
\hline $\mathrm{C}_{3} \times \mathrm{C}_{6}{ }^{5}$ & 2.43 & 2.14 & 0.70 & 2.27 & 2.55 & 0.65 \\
\hline $\mathrm{C}_{3}^{2} \times \mathrm{E}$ & 1.68 & 1.68 & 1.68 & 1.73 & 1.74 & 1.73 \\
\hline $\mathrm{C}_{3}^{2} \times \mathrm{C}_{6}$ & 2.14 & 0.70 & 2.43 & 0.64 & 2.27 & 2.55 \\
\hline $\mathrm{C}_{3}{ }^{2} \times \mathrm{C}_{3}$ & 2.67 & 1.21 & 2.71 & 1.30 & 2.85 & 2.90 \\
\hline $\mathrm{C}_{3}^{2} \times \mathrm{C}_{2}$ & 2.82 & 2.18 & 2.40 & 2.35 & 2.99 & 2.62 \\
\hline $\mathrm{C}_{3}{ }^{2} \times \mathrm{C}_{3}{ }^{2}$ & 2.49 & 2.66 & 1.64 & 2.85 & 2.61 & 1.82 \\
\hline $\mathrm{C}_{3}{ }^{2} \times \mathrm{C}_{6}^{5}$ & 1.91 & 2.47 & 1.11 & 2.61 & 1.96 & 1.18 \\
\hline
\end{tabular}


Table S3. R-Squared values of best fits for each combination of rotations considered for LF1.

\begin{tabular}{|c|c|c|c|c|c|c|c|c|c|c|c|c|}
\hline & $\begin{array}{l}\text { FA_C } \\
6 \_N_{-} \\
\text {Center } \\
+ \text { MA_ } \\
\text { C3 }\end{array}$ & $\begin{array}{l}\text { FA_C } \\
6 \_N_{-} \\
\text {Center } \\
+ \text { MA_ } \\
\text { total }\end{array}$ & $\begin{array}{l}\text { FA_C } \\
6 \_N_{-} \\
\text {Center }\end{array}$ & $\begin{array}{l}\text { FA_C } \\
\text { 6_Rot } \\
\text { ation+ } \\
\text { MA_C } \\
3\end{array}$ & $\begin{array}{l}\text { FA_C } \\
\text { 6_Rot } \\
\text { ation+ } \\
\text { MA_t } \\
\text { otal }\end{array}$ & $\begin{array}{l}\text { FA_C } \\
\text { 6_Rot } \\
\text { ation }\end{array}$ & $\begin{array}{l}\text { FA_C } \\
6 \_C- \\
\text { Center } \\
+ \text { MA_ } \\
\text { C3 }\end{array}$ & $\begin{array}{l}\text { FA_C } \\
6 \_C_{-} \\
\text {Center } \\
+ \text { MA_ } \\
\text { total }\end{array}$ & $\begin{array}{l}\text { FA_C } \\
\text { 6_C_ } \\
\text { Center }\end{array}$ & $\begin{array}{l}\text { FA_C } \\
\text { 6_Cen } \\
\text { termas } \\
\text { s_Cent } \\
\text { er+M } \\
\text { A_C3 }\end{array}$ & $\begin{array}{l}\text { FA_C } \\
\text { 6_Cen } \\
\text { termas } \\
\text { s_Cent } \\
\text { er+M } \\
\text { A_tota } \\
1\end{array}$ & $\begin{array}{l}\text { FA_C } \\
\text { 6_Cen } \\
\text { termas } \\
\text { s_Cent } \\
\text { er }\end{array}$ \\
\hline $\begin{array}{l}0 \mathrm{Br}- \\
80 \mathrm{~K}\end{array}$ & 0.984 & 0.986 & 0.984 & 0.987 & 0.953 & 0.904 & 0.985 & 0.966 & 0.966 & 0.984 & 0.985 & 0.984 \\
\hline $\begin{array}{l}10 \mathrm{Br}- \\
80 \mathrm{~K}\end{array}$ & 0.988 & 0.979 & 0.979 & 0.988 & 0.889 & 0.819 & 0.988 & 0.91 & 0.91 & 0.988 & 0.973 & 0.973 \\
\hline $\begin{array}{l}15 \mathrm{Br}- \\
80 \mathrm{~K}\end{array}$ & 0.991 & 0.991 & 0.991 & 0.989 & 0.932 & 0.878 & 0.988 & 0.949 & 0.949 & 0.987 & 0.986 & 0.986 \\
\hline $\begin{array}{l}20 \mathrm{Br}- \\
80 \mathrm{~K}\end{array}$ & 0.981 & 0.972 & 0.972 & 0.981 & 0.873 & 0.802 & 0.981 & 0.896 & 0.896 & 0.981 & 0.963 & 0.963 \\
\hline $\begin{array}{l}0 \mathrm{Br}- \\
100 \mathrm{~K}\end{array}$ & 0.988 & 0.994 & 0.988 & 0.995 & 0.971 & 0.929 & 0.994 & 0.982 & 0.982 & 0.989 & 0.993 & 0.989 \\
\hline $\begin{array}{l}10 \mathrm{Br}- \\
100 \mathrm{~K}\end{array}$ & 0.983 & 0.977 & 0.977 & 0.983 & 0.884 & 0.818 & 0.983 & 0.906 & 0.906 & 0.983 & 0.969 & 0.969 \\
\hline $\begin{array}{l}15 \mathrm{Br}- \\
100 \mathrm{~K}\end{array}$ & 0.987 & 0.987 & 0.987 & 0.985 & 0.936 & 0.885 & 0.984 & 0.953 & 0.953 & 0.984 & 0.983 & 0.983 \\
\hline $\begin{array}{l}20 \mathrm{Br}- \\
100 \mathrm{~K}\end{array}$ & 0.99 & 0.99 & 0.99 & 0.988 & 0.932 & 0.878 & 0.987 & 0.949 & 0.949 & 0.987 & 0.986 & 0.986 \\
\hline $\begin{array}{l}\mathrm{Br}- \\
120 \mathrm{~K} \\
\end{array}$ & 0.981 & 0.991 & 0.981 & 0.993 & 0.967 & 0.939 & 0.991 & 0.984 & 0.984 & 0.982 & 0.99 & 0.982 \\
\hline $\begin{array}{l}10 \mathrm{Br}- \\
120 \mathrm{~K}\end{array}$ & 0.983 & 0.983 & 0.983 & 0.975 & 0.925 & 0.881 & 0.978 & 0.947 & 0.947 & 0.978 & 0.978 & 0.978 \\
\hline $\begin{array}{l}15 \mathrm{Br}- \\
120 \mathrm{~K}\end{array}$ & 0.976 & 0.979 & 0.976 & 0.98 & 0.948 & 0.911 & 0.977 & 0.963 & 0.963 & 0.974 & 0.975 & 0.974 \\
\hline $\begin{array}{l}20 \mathrm{Br}- \\
120 \mathrm{~K}\end{array}$ & 0.987 & 0.989 & 0.987 & 0.987 & 0.95 & 0.91 & 0.987 & 0.968 & 0.968 & 0.986 & 0.986 & 0.986 \\
\hline $\begin{array}{l}\mathrm{BBr}- \\
160 \mathrm{~K} \\
\end{array}$ & 0.977 & 0.978 & 0.977 & 0.961 & 0.928 & 0.904 & 0.974 & 0.958 & 0.958 & 0.974 & 0.975 & 0.974 \\
\hline $\begin{array}{l}10 \mathrm{Br}- \\
160 \mathrm{~K}\end{array}$ & 0.959 & 0.96 & 0.959 & 0.945 & 0.906 & 0.879 & 0.954 & 0.934 & 0.934 & 0.955 & 0.955 & 0.955 \\
\hline $\begin{array}{l}15 \mathrm{Br}- \\
160 \mathrm{~K}\end{array}$ & 0.95 & 0.961 & 0.95 & 0.965 & 0.941 & 0.922 & 0.961 & 0.956 & 0.956 & 0.949 & 0.958 & 0.949 \\
\hline $\begin{array}{l}20 \mathrm{Br}- \\
160 \mathrm{~K}\end{array}$ & 0.953 & 0.952 & 0.952 & 0.921 & 0.884 & 0.847 & 0.945 & 0.919 & 0.919 & 0.954 & 0.953 & 0.953 \\
\hline
\end{tabular}


Table S4. R-Squared values of best fits for each combination of rotations considered for LF2.

\begin{tabular}{|c|c|c|c|c|c|c|c|c|c|c|c|c|}
\hline & $\begin{array}{l}\text { FA_C } \\
6 \_N_{-} \\
\text {Center } \\
+\mathrm{MA} \\
\mathrm{C} 3\end{array}$ & $\begin{array}{l}\text { FA_C } \\
6 \_N_{-} \\
\text {Center } \\
\text { +MA_ } \\
\text { total }\end{array}$ & $\begin{array}{l}\text { FA_C } \\
6 \_N_{-} \\
\text {Center }\end{array}$ & $\begin{array}{l}\text { FA_C } \\
\text { 6_Rot } \\
\text { ation+ } \\
\text { MA_C } \\
3\end{array}$ & $\begin{array}{l}\text { FA_C } \\
\text { 6_Rot } \\
\text { ation+ } \\
\text { MA_t } \\
\text { otal }\end{array}$ & $\begin{array}{l}\text { FA_C } \\
\text { 6_Rot } \\
\text { ation }\end{array}$ & $\begin{array}{l}\text { FA_C } \\
6 \_C- \\
\text { Center } \\
+ \text { MA_ } \\
\text { C3 }\end{array}$ & $\begin{array}{l}\text { FA_C } \\
\text { 6_C_ } \\
\text { Center } \\
\text { +MA_ } \\
\text { total }\end{array}$ & $\begin{array}{l}\text { FA_C } \\
\text { 6_C_ } \\
\text { Center }\end{array}$ & $\begin{array}{l}\text { FA_C } \\
\text { 6_Cen } \\
\text { termas } \\
\text { s_Cent } \\
\text { er+M } \\
\text { A_C3 }\end{array}$ & $\begin{array}{l}\text { FA_C } \\
\text { 6_Cen } \\
\text { termas } \\
\text { s_Cent } \\
\text { er+M } \\
\text { A_tota } \\
1\end{array}$ & $\begin{array}{l}\text { FA_C } \\
\text { 6_Cen } \\
\text { termas } \\
\text { s_Cent } \\
\text { er }\end{array}$ \\
\hline $\begin{array}{l}0 \mathrm{Br}- \\
80 \mathrm{~K}\end{array}$ & 0.904 & 0.928 & 0.904 & 0.938 & 0.916 & 0.897 & 0.93 & 0.928 & 0.928 & 0.904 & 0.925 & 0.904 \\
\hline $\begin{array}{l}10 \mathrm{Br}- \\
80 \mathrm{~K}\end{array}$ & 0.893 & 0.926 & 0.893 & 0.935 & 0.921 & 0.903 & 0.929 & 0.929 & 0.929 & 0.896 & 0.925 & 0.896 \\
\hline $\begin{array}{l}15 \mathrm{Br}- \\
80 \mathrm{~K}\end{array}$ & 0.968 & 0.972 & 0.968 & 0.973 & 0.942 & 0.898 & 0.971 & 0.956 & 0.956 & 0.967 & 0.97 & 0.967 \\
\hline $\begin{array}{l}20 \mathrm{Br}- \\
80 \mathrm{~K}\end{array}$ & 0.955 & 0.985 & 0.955 & 0.986 & 0.982 & 0.956 & 0.986 & 0.986 & 0.986 & 0.961 & 0.985 & 0.961 \\
\hline $\begin{array}{l}0 \mathrm{Br}- \\
100 \mathrm{~K}\end{array}$ & 0.886 & 0.936 & 0.886 & 0.952 & 0.939 & 0.931 & 0.941 & 0.941 & 0.941 & 0.889 & 0.935 & 0.889 \\
\hline $\begin{array}{l}10 \mathrm{Br}- \\
100 \mathrm{~K}\end{array}$ & 0.956 & 0.971 & 0.956 & 0.975 & 0.956 & 0.927 & 0.971 & 0.967 & 0.967 & 0.957 & 0.969 & 0.957 \\
\hline $\begin{array}{l}15 \mathrm{Br}- \\
100 \mathrm{~K}\end{array}$ & 0.929 & 0.956 & 0.929 & 0.964 & 0.948 & 0.931 & 0.958 & 0.957 & 0.957 & 0.93 & 0.954 & 0.93 \\
\hline $\begin{array}{l}20 \mathrm{Br}- \\
100 \mathrm{~K}\end{array}$ & 0.932 & 0.98 & 0.932 & 0.982 & 0.979 & 0.962 & 0.981 & 0.981 & 0.981 & 0.94 & 0.979 & 0.94 \\
\hline $\begin{array}{l}\mathrm{Br}- \\
120 \mathrm{~K}\end{array}$ & 0.946 & 0.979 & 0.946 & 0.984 & 0.975 & 0.953 & 0.981 & 0.981 & 0.981 & 0.951 & 0.978 & 0.951 \\
\hline $\begin{array}{l}10 \mathrm{Br}- \\
120 \mathrm{~K}\end{array}$ & 0.932 & 0.956 & 0.932 & 0.963 & 0.948 & 0.927 & 0.958 & 0.957 & 0.957 & 0.934 & 0.954 & 0.934 \\
\hline $\begin{array}{l}15 \mathrm{Br}- \\
120 \mathrm{~K}\end{array}$ & 0.952 & 0.975 & 0.952 & 0.98 & 0.968 & 0.942 & 0.977 & 0.975 & 0.975 & 0.955 & 0.974 & 0.955 \\
\hline $\begin{array}{l}20 \mathrm{Br}- \\
120 \mathrm{~K}\end{array}$ & 0.926 & 0.979 & 0.926 & 0.978 & 0.979 & 0.962 & 0.979 & 0.979 & 0.979 & 0.936 & 0.979 & 0.936 \\
\hline $\begin{array}{l}\text { OBr- } \\
160 \mathrm{~K}\end{array}$ & 0.905 & 0.954 & 0.905 & 0.978 & 0.974 & 0.968 & 0.97 & 0.971 & 0.97 & 0.913 & 0.955 & 0.913 \\
\hline $\begin{array}{l}10 \mathrm{Br}- \\
160 \mathrm{~K}\end{array}$ & 0.917 & 0.957 & 0.917 & 0.966 & 0.959 & 0.948 & 0.962 & 0.962 & 0.962 & 0.922 & 0.957 & 0.922 \\
\hline $\begin{array}{l}15 \mathrm{Br}- \\
160 \mathrm{~K}\end{array}$ & 0.907 & 0.962 & 0.907 & 0.972 & 0.968 & 0.961 & 0.966 & 0.966 & 0.966 & 0.914 & 0.961 & 0.914 \\
\hline $\begin{array}{l}20 \mathrm{Br}- \\
160 \mathrm{~K}\end{array}$ & 0.925 & 0.972 & 0.925 & 0.969 & 0.974 & 0.951 & 0.975 & 0.978 & 0.975 & 0.938 & 0.974 & 0.938 \\
\hline
\end{tabular}



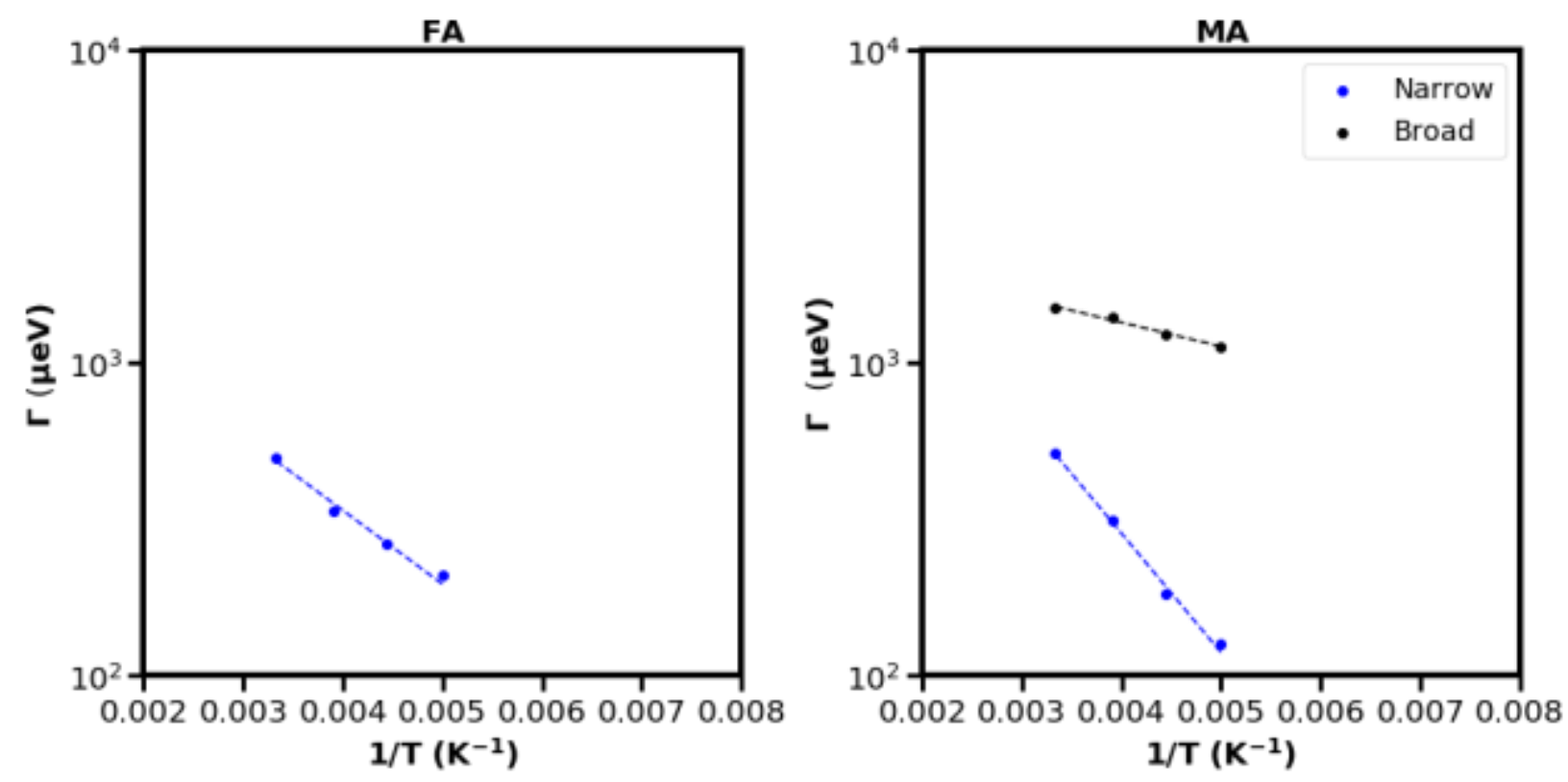

Figure S7. Arrhenius fits of pure $\mathrm{FAPbBr}$ (left) and pure $\mathrm{MAPbBr}$ (right)

Table S5: Activation Energies of FA and MA in $\mathrm{FAPbBr}$ and $\mathrm{MAPbBr}$, respectively

\begin{tabular}{|l|l|}
\hline Rotation & Activation Energy $(\mathrm{meV})$ \\
\hline $\mathrm{FA} \mathrm{C}_{6}$ & $47+/-4$ \\
\hline $\mathrm{MA} \mathrm{C}_{3}$ & $15+/-3$ \\
\hline MA C $_{6}$ & $76+/-1$ \\
\hline
\end{tabular}



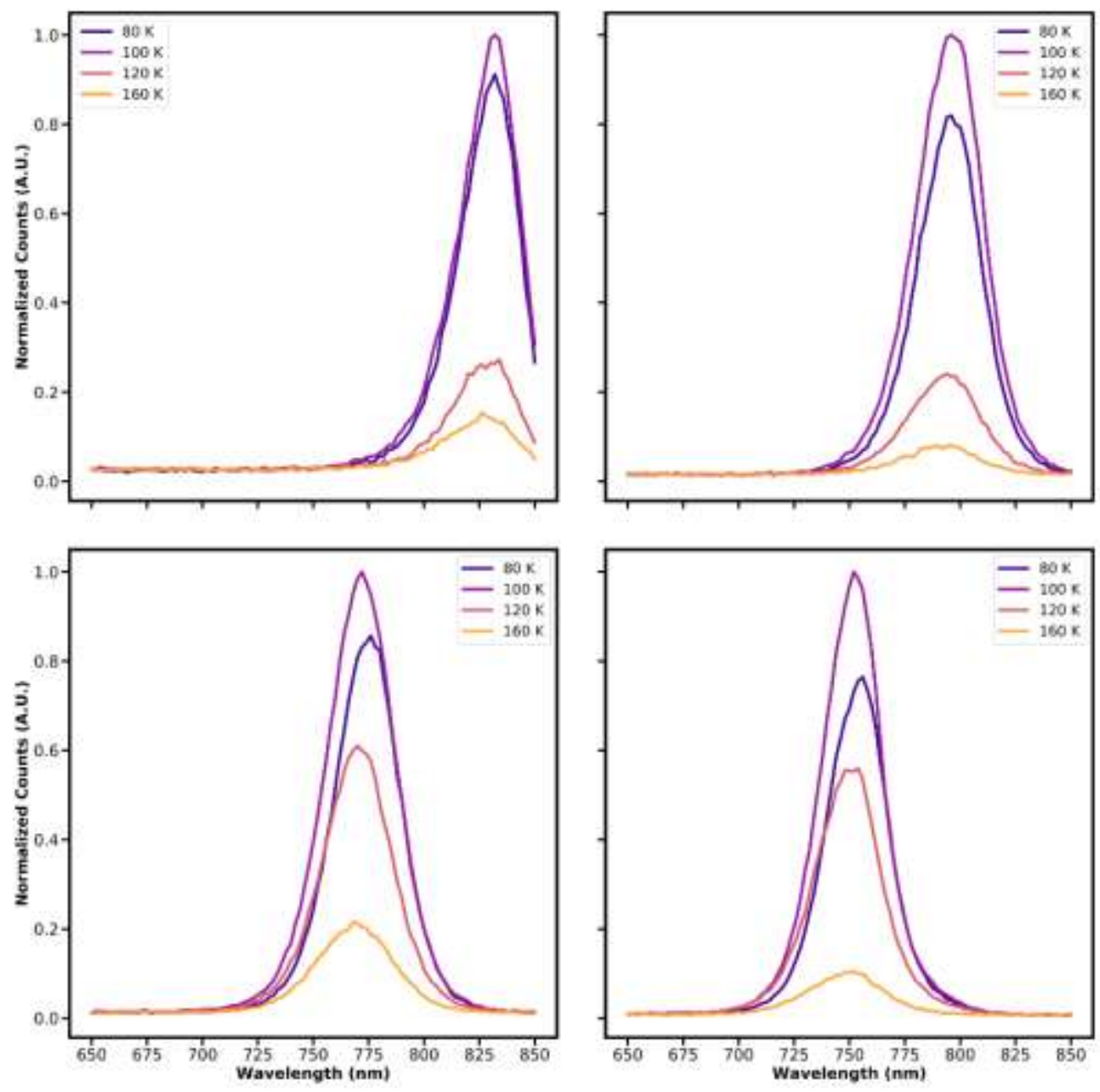

Figure S8. Low temperature PL spectra of each crystals containing $0 \% \mathrm{Br}$ (top left), $10 \% \mathrm{Br}$ (top right), $15 \% \mathrm{Br}$ (bottom left) and $20 \% \mathrm{Br}$ (bottom right). 

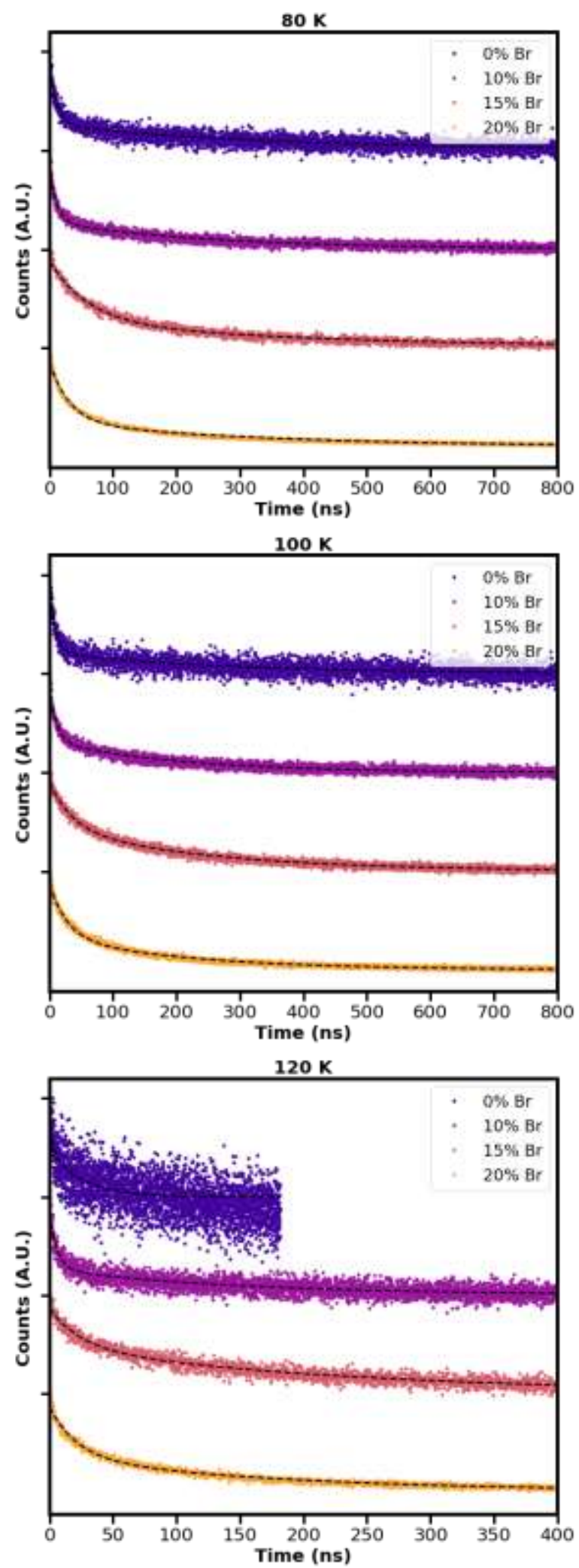

Figure S9. Low temperature PL lifetimes of each of the four samples at different temperatures. At $120 \mathrm{~K}$, the signal from the $0 \% \mathrm{Br}$ sample needed to be measured with a $200 \mathrm{~ns}$ pulse in order to obtain signal above the noise floor. 


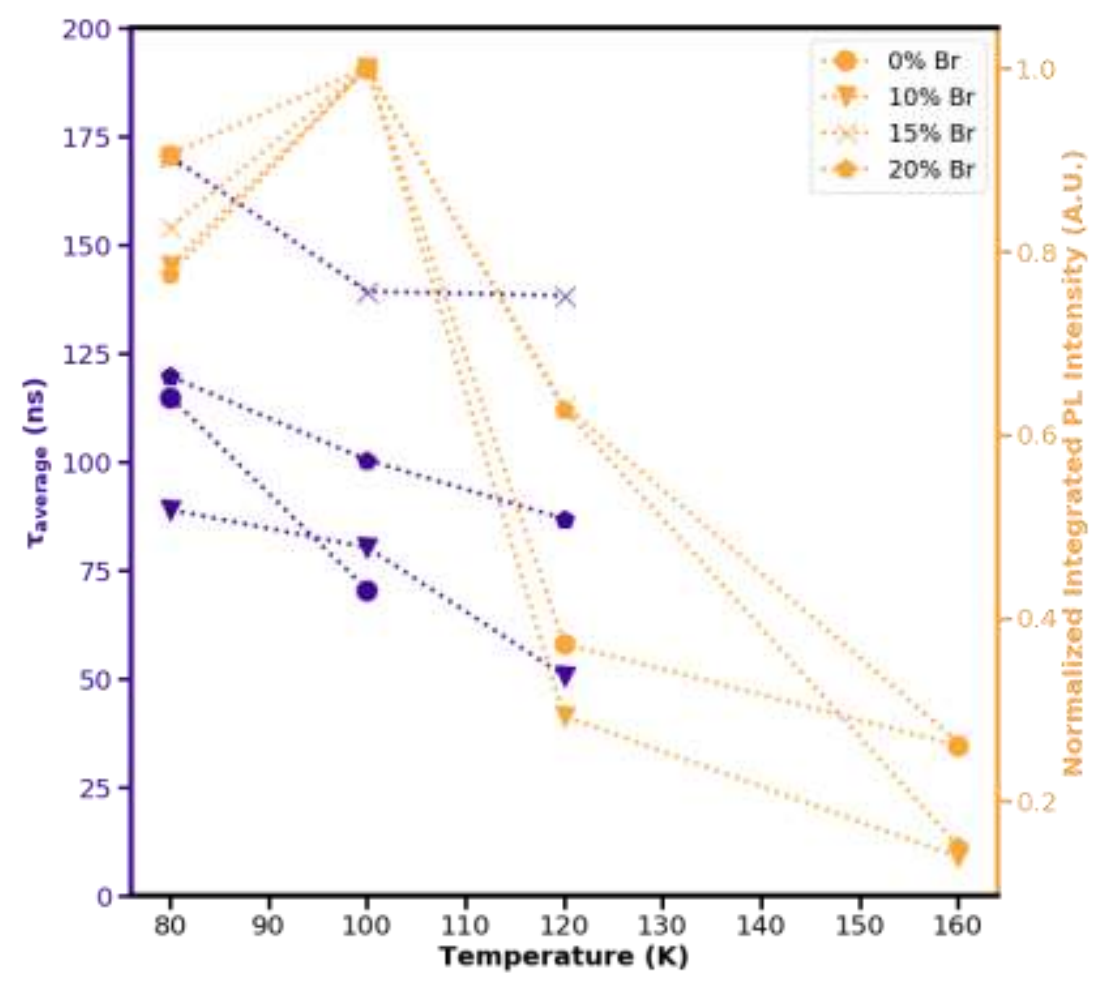

Figure S10. Average PL lifetimes of each of the four samples as a function of temperature (left) and integrated intensities, relative to the maximum intensity for each sample (right) 


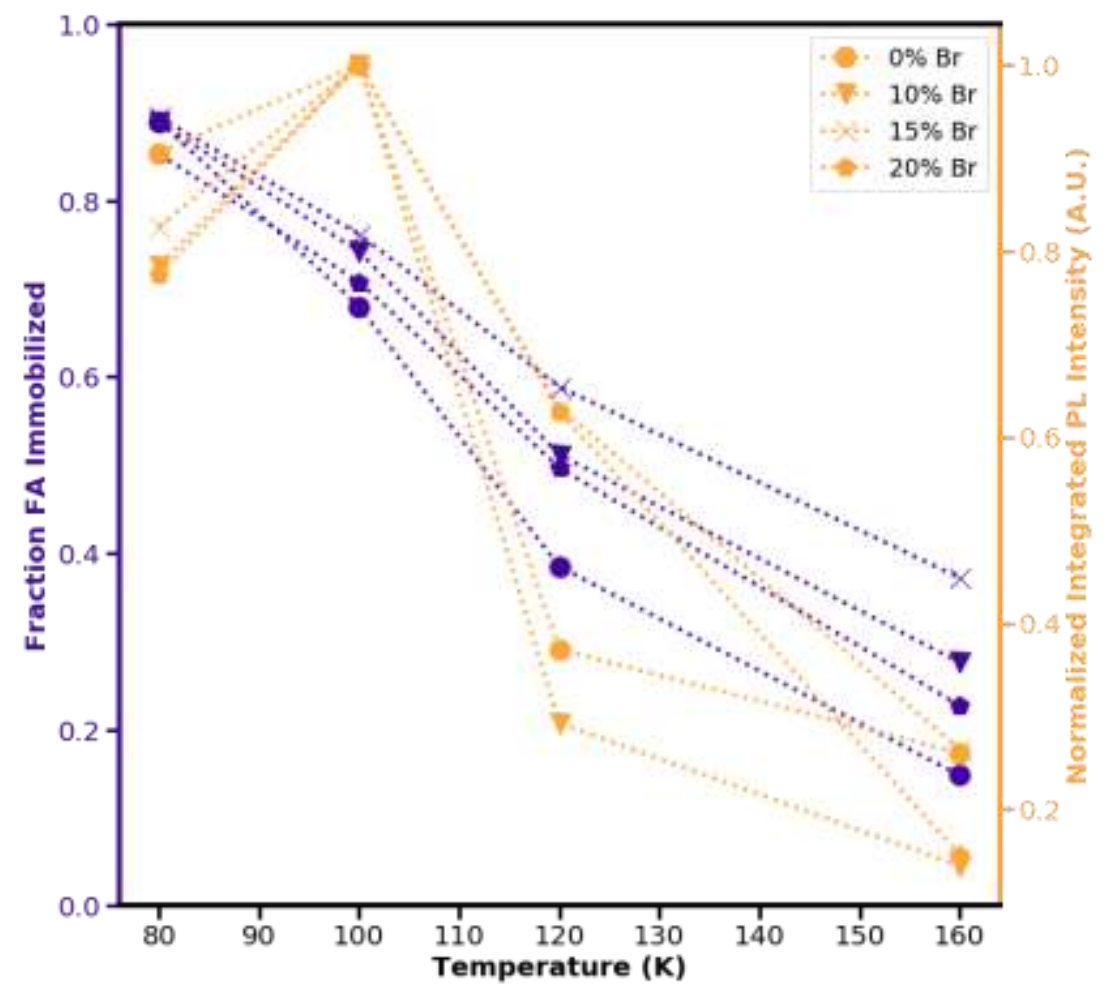

Figure S11. Fraction of immobilized FA and the integrated PL intensity of each sample as a function of temperature. 


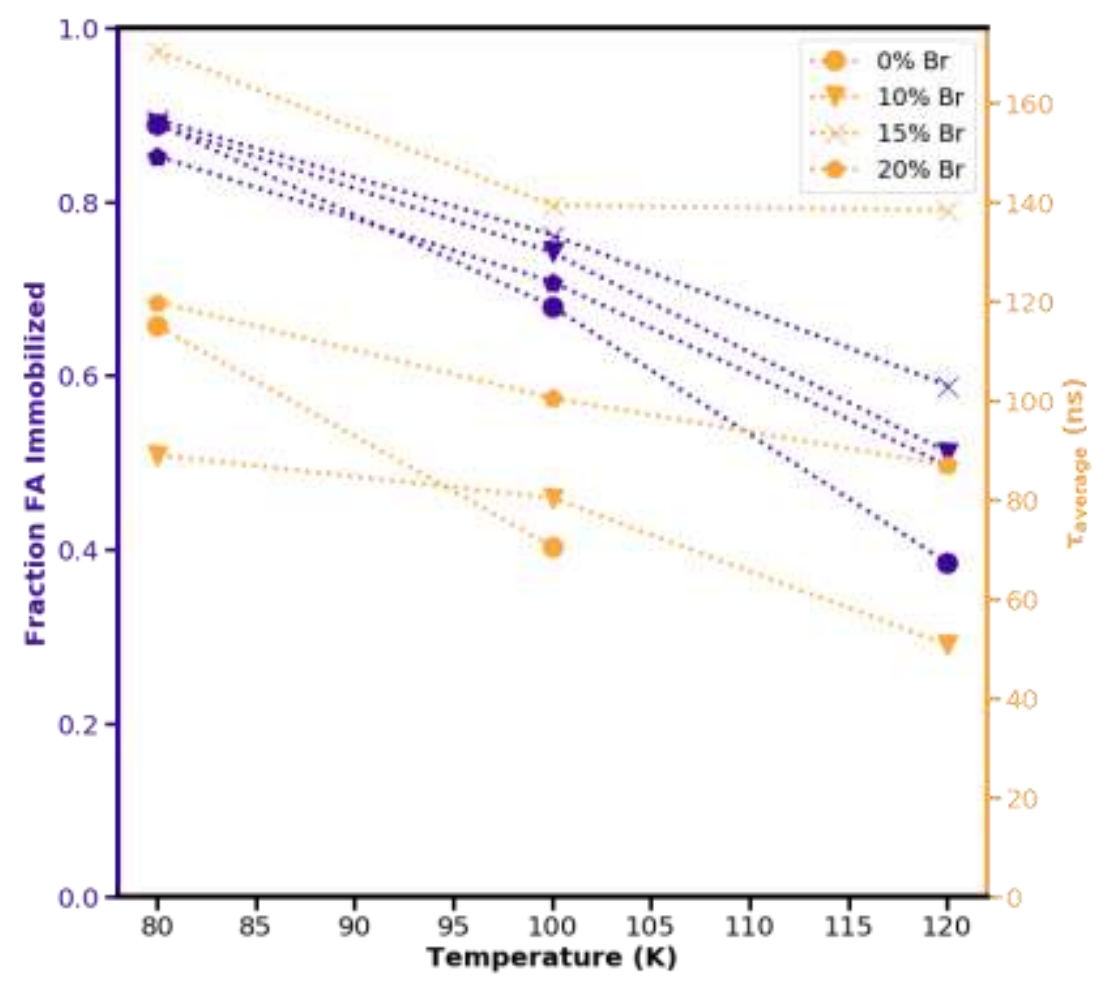

Figure S12. Fraction of immobilized FA and the average PL lifetime of each sample as a function of temperature. 

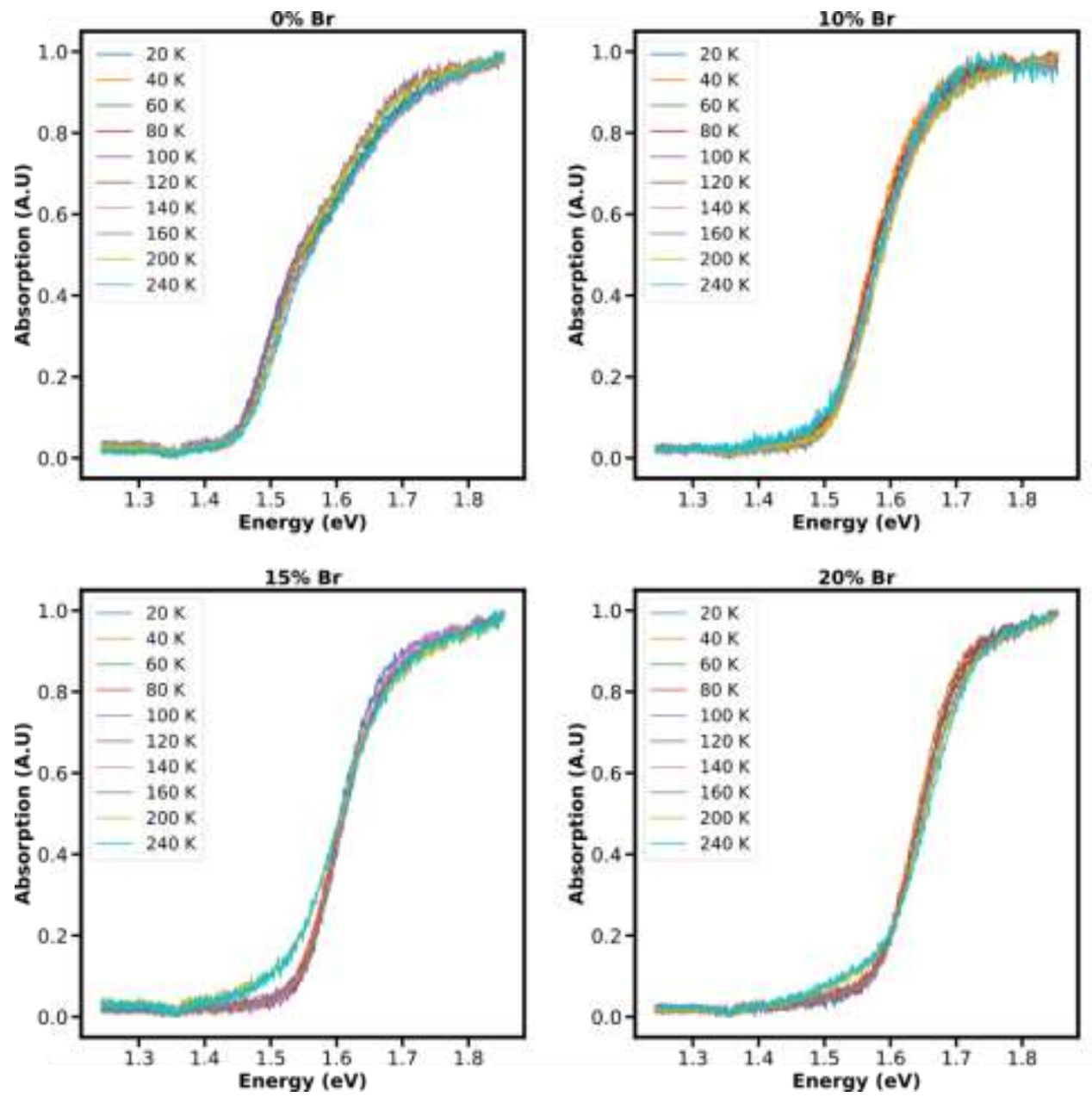

Figure S13. Low-temperature absorption spectra of each sample, shown at different temperatures.

Table S6. Extracted Exciton binding energies for each sample at each temperature (meV).

\begin{tabular}{|l|l|l|l|l|}
\hline & $0 \% \mathrm{Br}$ & $10 \% \mathrm{Br}$ & $15 \% \mathrm{Br}$ & $20 \% \mathrm{Br}$ \\
\hline $20 \mathrm{~K}$ & 1.33 & 1.31 & 5.74 & 2.65 \\
\hline $40 \mathrm{~K}$ & 1.20 & 1.62 & 5.40 & 1.91 \\
\hline $60 \mathrm{~K}$ & 1.50 & 1.48 & 4.55 & 1.86 \\
\hline $80 \mathrm{~K}$ & 1.31 & 1.53 & 4.71 & 1.62 \\
\hline $100 \mathrm{~K}$ & 1.52 & 1.25 & 4.12 & 1.73 \\
\hline $120 \mathrm{~K}$ & 2.03 & 1.52 & 3.88 & 2.12 \\
\hline $140 \mathrm{~K}$ & 1.87 & 1.39 & 4.67 & 1.94 \\
\hline $160 \mathrm{~K}$ & 1.50 & 1.32 & 4.26 & 2.08 \\
\hline $200 \mathrm{~K}$ & 1.29 & 1.17 & 6.80 & 2.33 \\
\hline $240 \mathrm{~K}$ & 1.30 & 2.10 & 6.78 & 2.46 \\
\hline
\end{tabular}



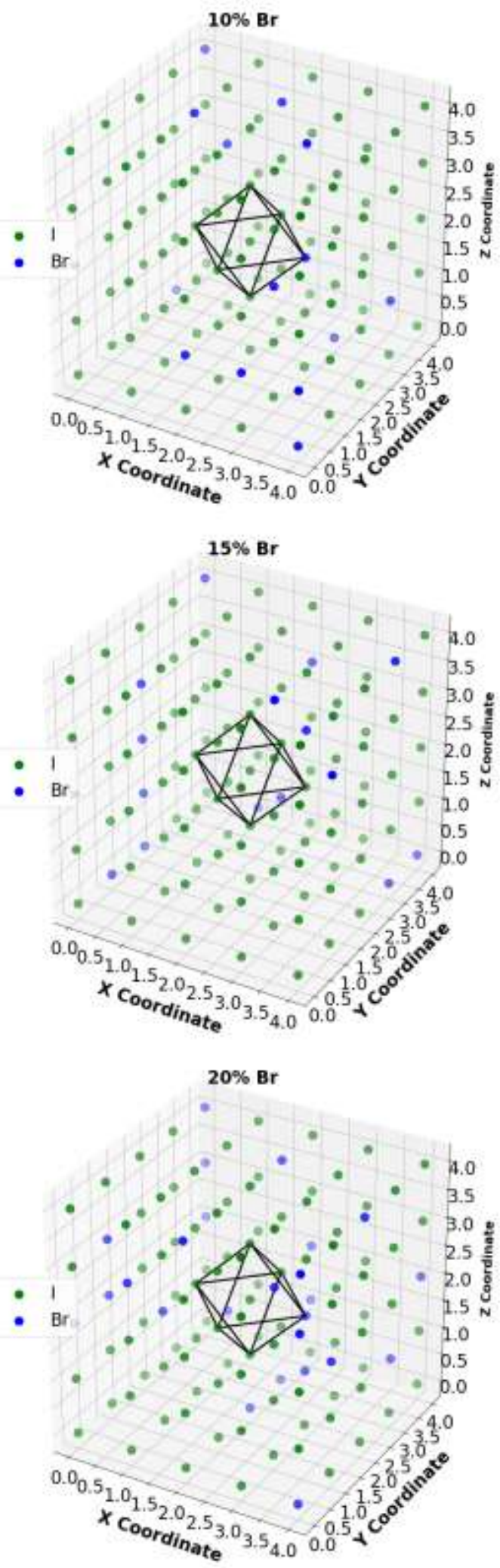

Figure S14. Monte Carlo simulation of bromine incorporation into perovskite unit cell. The solid lines represent an example octahedron for which the number of bromines is counted. 

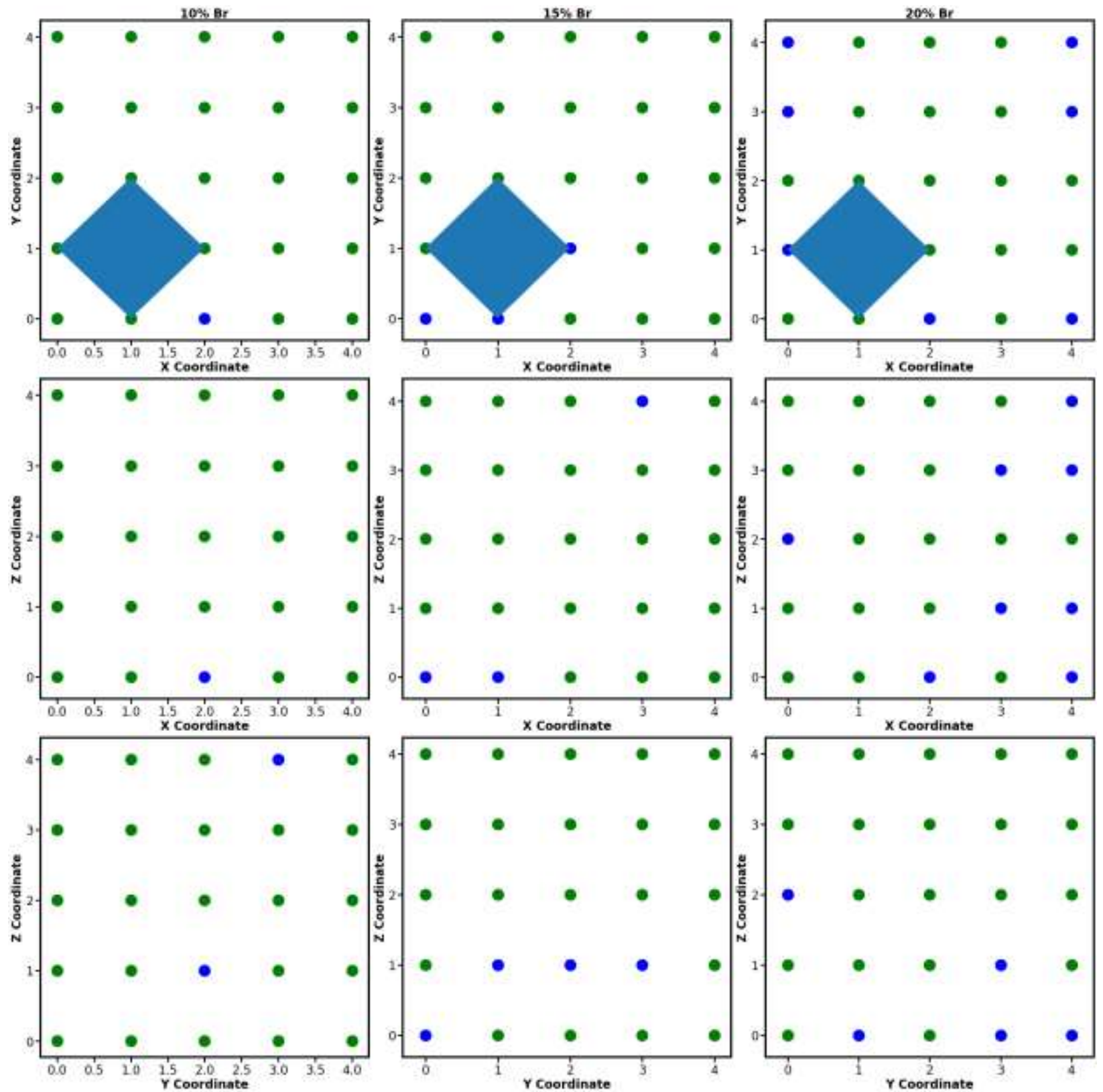

Figure S15: 2D Visualization of the simulated cells with different $\mathrm{Br}$ concentrations. Only the halogen atoms are shown. The solid shape indicates the top-down view of the octahedron used to calculate the number of bromine atoms present. 

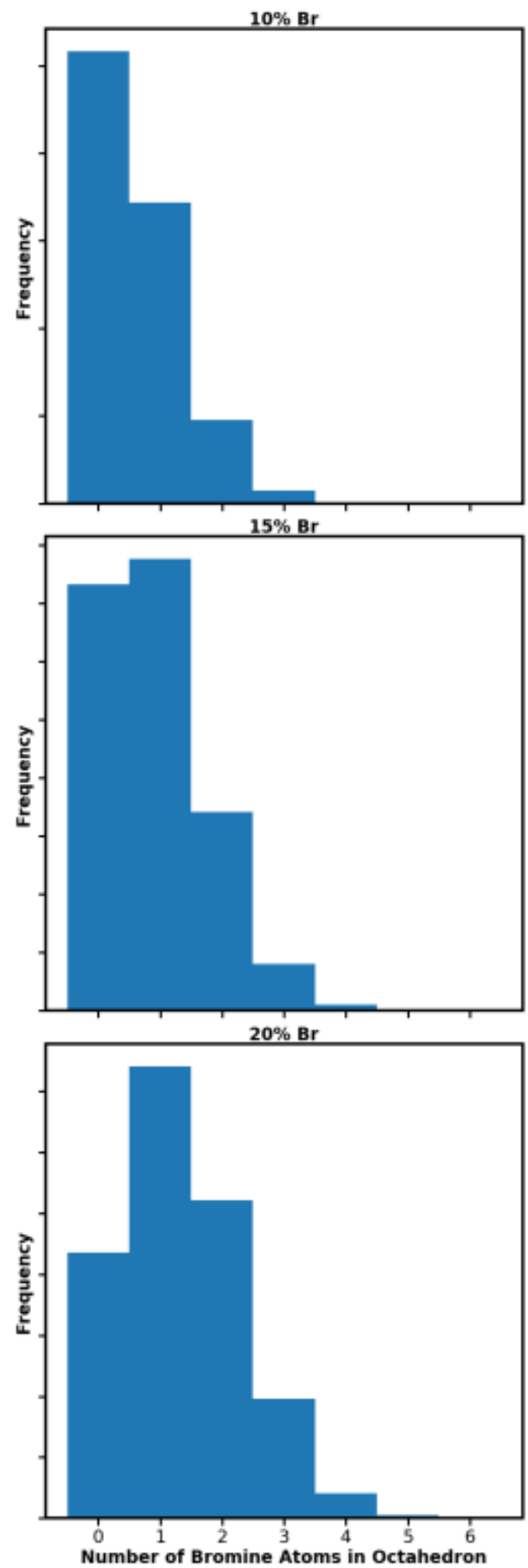

Figure S16: Distribution of number of Bromine atoms in each unit cell at different bromine incorporations. The simulation was performed on a $200 \times 200 \times 200$ supercell. The average number of $\mathrm{Br}$ atoms in an octahedron were evaluated to be $0.8,0.9$ and 1.2 for $10 \%, 15 \%$ and $20 \%$ incorporation respectively. 


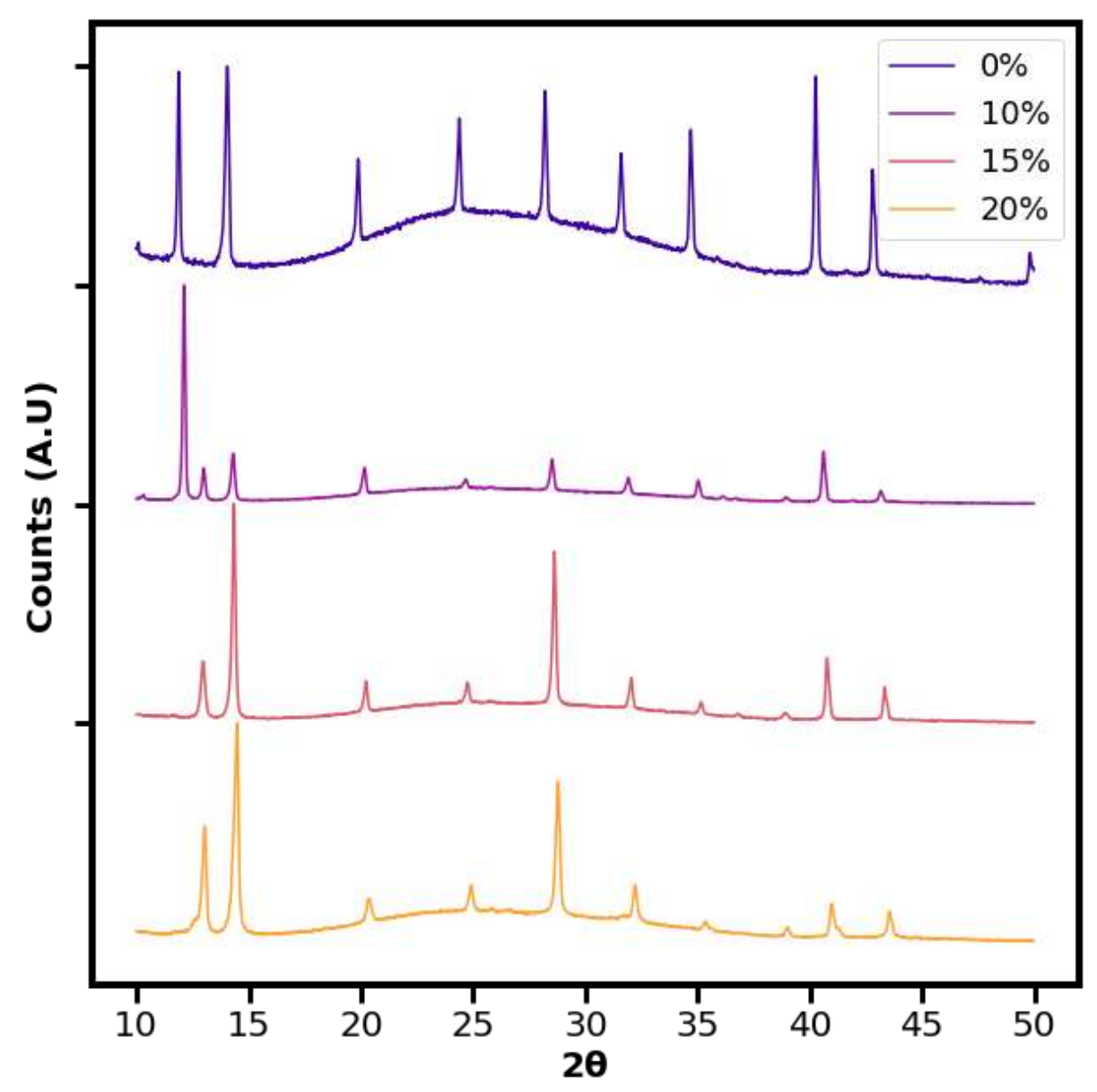

Figure S17. Powder XRD of thin films of the triple-cation mixed-halide perovskites with different bromine incorporations.

Table S7. Bromine:Iodine ratios of different thin-films, measured with XPS.

\begin{tabular}{|l|l|}
\hline Br:I in precursor solution & Br:I in final thin-film \\
\hline $0: 100$ & $0: 100$ \\
\hline $10: 90$ & $9.5: 90.5$ \\
\hline $15: 85$ & $13.5: 86.5$ \\
\hline $20: 80$ & $19: 81$ \\
\hline
\end{tabular}




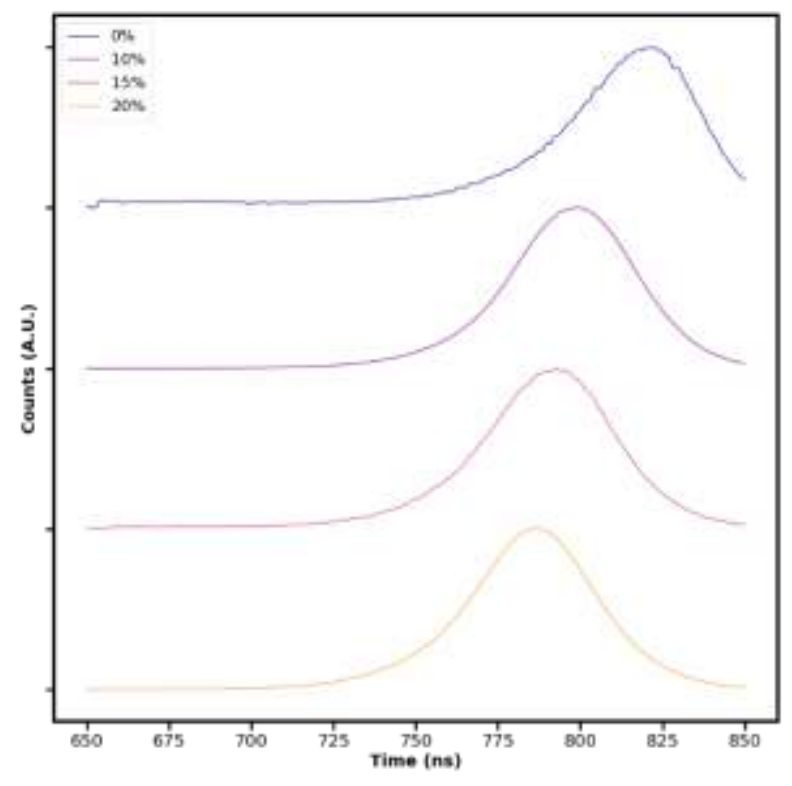

Figure S18. Steady-state PL spectra of the triple-cation mixed-halide perovskites with different bromine incorporations.
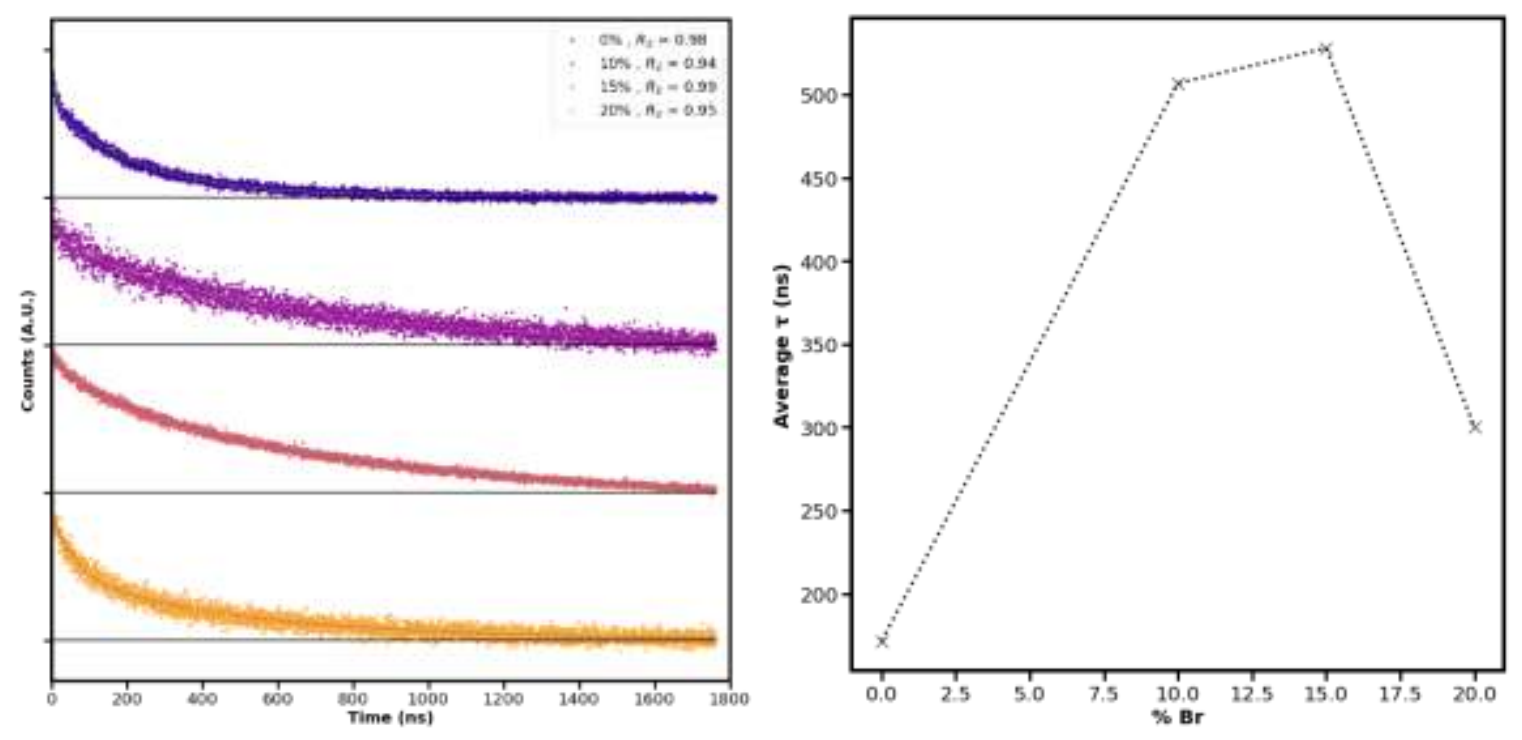

Figure S19. Transient PL spectra of each of the thin-film samples at room temperature (left) and the average lifetime extracted from the biexponential fit (right). 
Table S8. Experimental results of antisolvent method growth of tripled-cation mixed-halide single crystals.

\begin{tabular}{|l|r|l|l|}
\hline Antisolvent & $\begin{array}{l}\text { Concentration (Pb } \\
\text { M) }\end{array}$ & $\begin{array}{l}\text { Crystals } \\
\text { Grown }\end{array}$ & Notes \\
\hline Dichloromethane & 1 & No & $\begin{array}{l}\text { White } \\
\text { precipitate }\end{array}$ \\
\hline Chloroform & 1 & No & $\begin{array}{l}\text { White } \\
\text { precipitate }\end{array}$ \\
\hline Chlorobenzene & 1 & Yes & $\begin{array}{l}\text { Multi-phased, } \\
\text { yellow colour }\end{array}$ \\
\hline Toluene & 1 & No & \\
\hline Ethyl Acetate & 1 & No & \\
\hline Dichloromethane & 0.67 & No & $\begin{array}{l}\text { White } \\
\text { precipitate }\end{array}$ \\
\hline Chloroform & 0.67 & No & \\
\hline Chlorobenzene & 0.67 & No & \\
\hline Toluene & 0.67 & No & \\
\hline Ethyl Acetate & 0.67 & No & \\
\hline Dichloromethane & 1.5 & No & \\
\hline Chloroform & 1.5 & No & \\
\hline Chlorobenzene & 1.5 & Yes & $\begin{array}{l}\text { Multi-phased, } \\
\text { yellow colour }\end{array}$ \\
\hline
\end{tabular}

References

(1) Chen, T.; Foley, B. J.; Park, C.; Brown, C. M.; Harriger, L. W.; Lee, J.; Ruff, J.; Yoon, M.; Choi, J. J.; Lee, S.-H. Entropy-Driven Structural Transition and Kinetic Trapping in Formamidinium Lead Iodide Perovskite. Sci. Adv. 2016, 7. 